\title{
Change of impact kind in vibroimpact system due its parameters changing
}

\author{
V.A. Bazhenov ${ }^{1}$, O.S. Pogorelova ${ }^{1}$, and T.G. Postnikova ${ }^{1}$ \\ ${ }^{1}$ Kyiv National University of Construction and Architecture, Ukraine
}

\begin{abstract}
The possibility of contact kind changing by system parameters changing is researching. It is investigated the contact between bodies in two-mass two-degree-of-freedom vibroimpact system. We show which parameters changing can transform the rigid impact in system into the soft one. In the main these parameters are the Young's modulus and geometrical characteristics of the contact zone for both bodies.
\end{abstract}

\section{Introduction}

Vibroimpact machines and equipment are often encountered in many applications of engineering practice. In general systems in which impacts of matching elements occur play an important role in the theory of vibration of mechanical systems. Therefore the study of their dynamic behaviour and vibroimpact movement characteristics in different functional condition has got the special interest. Such investigations are developed extensively during the last decades [1-3].

There is the vibroimpact systems (VIS) classifications by different aspects [4]. One of them is impact kind characteristic - rigid or soft. Some principal difference between rigid and soft contact were formulated in [5]. The main sign is its duration. Just impact duration dictates the way of its simulation. If impact duration is large impact isn't instantaneous. Its simulation by boundary conditions with Newton's restitution coefficient using based on stereomechanic theory isn't possible [6]. The stiffness of VIS elements causes the impact softness.

The soft impacts take place in engineering very often. The authors write in [7]: "Soft impacts occur in many practical mechanical systems where there is some "cushioning" at the impacting surfaces - meant for reducing the noise and chatter. It can be visualized as a mass impacting not with a hard wall, but with a springdamper support in front of a wall. The existence of the spring-damper type cushion introduces some special features in the system dynamics".

The investigation of systems which constituted by softly impacting beams and rods of non-negligible mass is fulfilled in [8]. The impact is simulated by force which depends linearly from bodies penetrations one into another. Some virtual spring imitates this force. The values of bodies' displacements and penetrations characterize the impact softness. The authors investigate the influence of virtual spring stiffness at system dynamic behaviour and vibratory motion character.

In our works we studied two models of VIS. The first model is VIS with rigid impact. Its calculation scheme is corresponding to the system with percussive or nonpercussive dynamic damper [9] (figure 1).

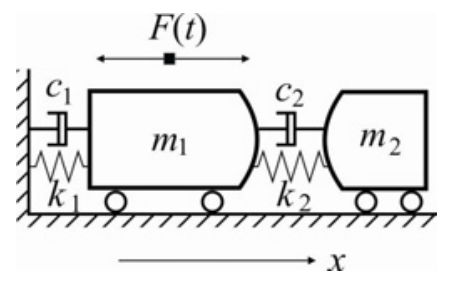

Fig. 1. Model of VIS with rigid impact.

Another model is VIS with soft impact. Its calculation scheme is corresponding to vibroimpact platform, which is widely used in building industry for concrete mix compaction and concrete products forming [6] (figure 2).

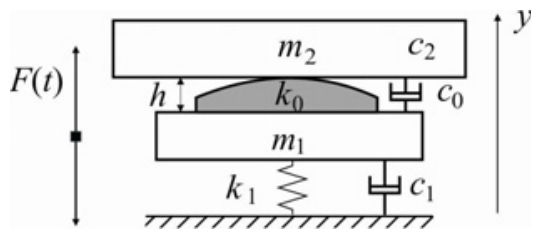

Fig. 2. Model of VIS with soft impact.

Contact impact forces in these systems in dependence from time have got the forms that are shown at figures 3 . Numerical parameters values have been written in $[6,9]$. The rigid impact is almost instantaneous because its duration is very small. Therefore the contact force graphic in every impact has the form of "stick". On the contrary the duration of soft impact is large; the contact force graphic in every impact has the form of "bell" [10]. It should be noted that the frequency of external loading 
at vibroimpact platform is larger to 20 times then the frequency of external loading at vibroimpact system with rigid impact. Therefore the scale of time axis at figures $3 \mathrm{a}, \mathrm{b}$ is different for these two models. We have shown that the picture of contact force for rigid impact fulfilled in the same time scale which have used for "bells" at figure 3b has not changed - "stick" have remained as "stick". Such comparison is more correct, pure.

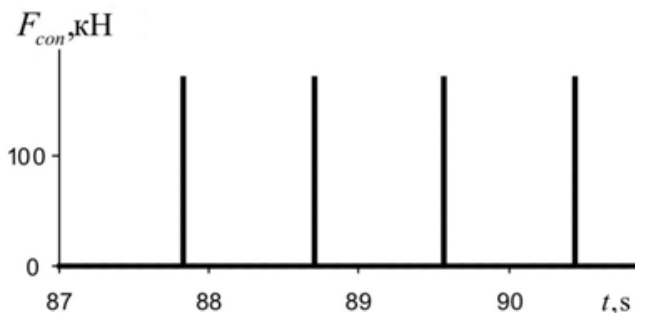

(a)

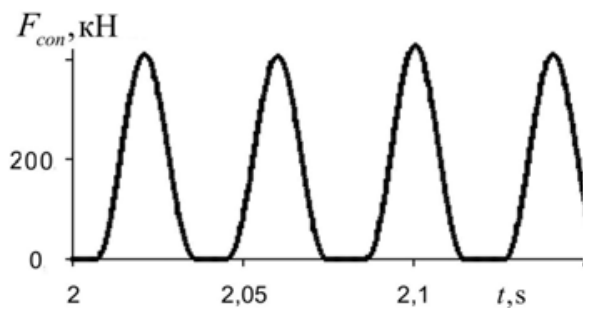

(b)

Fig. 3. Character of contact force exited in VIS: (a) - with rigid impact, (b) - with soft impact.

The bodies displacements in dependence from time are shown at figure 4.

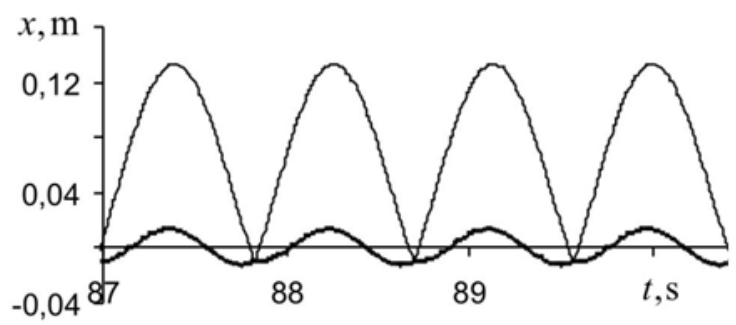

(a)

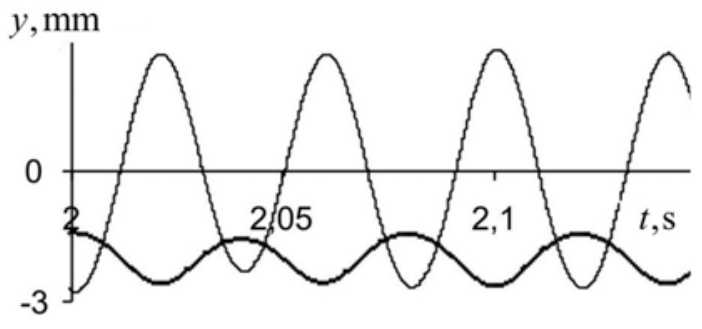

(b)

Fig. 4. Bodies' displacements in VIS: (a) - with rigid impact, (b) - with soft impact.

Let us look attentively at the figure 4 . For the system with rigid impact it is seen well as attached body (thin curve) jumps away from the main one (thick curve) in a moment. It doesn't penetrate into the main body. On the contrary in the system with soft impact the upper body when falling and shocking the lower one causes its considerable deformation and penetrates into it.

Is it possible to select the parameters of VIS with rigid impact like that the impact in it will become the soft one? This article searches the answer at this question.

\section{The main equations}

We'll study the problem about impact softening using the scheme of two-of-degree-freedom VIS of two bodies which is shown at figure 1. This VIS is described in [10]. Let us remind of it shortly. The system is formed by the main body and attached one. Bodies are connected by linear elastic springs and dampers. The main body is subjected to the action of periodical external force:

$$
F(t)=\lambda F_{0} \cos \left(\omega t+\varphi_{0}\right)
$$

where $\lambda$ is non-dimensional parameter of its intensity. We consider the elastic collinear impacts with low velocities. The contact surfaces are smooth, curvilinear, without roughness. For impact simulation we use the Hertz's contact interaction force based on quasistatic Hertz's theory [11]:

$$
\begin{aligned}
& F_{c o n}(z)=K[H(z) z(t)]^{3 / 2}, \\
& K=\frac{4}{3} \frac{q}{\left(\delta_{1}+\delta_{2}\right) \sqrt{A+B}}, \\
& \delta_{1}=\frac{1-\mu_{1}^{2}}{E_{1} \pi}, \delta_{2}=\frac{1-\mu_{2}^{2}}{E_{2} \pi},
\end{aligned}
$$

where $z(t)$ is the relative closing in of bodies due the local deformation in contact zone, $z(t)=x_{1}-x_{2}, H(z)$ is the Heaviside step function, $\mu_{i}$ and $E_{i}$ are respectively Poisson's ratios and Young's modulus for both bodies, $A$, $B$ and $q$ are the characteristics of contact zone local geometry. We consider that these surfaces are spherical, then $A=B=1 / 2 R_{1}+1 / 2 R_{2}$, where $R_{1}, R_{2}$ are the contact surfaces radiuses. The Hertz/s theory takes into account only local deformations in contact zone.

In [12] the authors examine the limitations for using of Hertz's theory in different individual cases. However the using of less rough impact models for VIS (for example the wave theory) causes considerable difficulties due to repeated impacts. Therefore Hertz's theory is widely used for analysis of VIS dynamics now too.

Movement equations of the system have the form:

$$
\begin{aligned}
& \ddot{x}_{1}=-2 \xi_{1} \omega_{1} \dot{x}_{1}-\omega_{1}^{2} x_{1}-2 \xi_{2} \omega_{2} \chi\left(\dot{x}_{1}-\dot{x}_{2}\right)- \\
& \omega_{2}^{2} \chi\left(x_{1}-x_{2}+D\right)+ \\
& \frac{1}{m_{1}}\left[F(t)-F_{c o n}\left(x_{1}-x_{2}\right)\right], \\
& \ddot{x}_{2}=-2 \xi_{2} \omega_{2}\left(\dot{x}_{2}-\dot{x}_{1}\right)- \\
& \omega_{2}^{2}\left(x_{2}-x_{1}-D\right)+\frac{1}{m_{2}} F_{c o n}\left(x_{1}-x_{2}\right),
\end{aligned}
$$


where $\quad \omega_{1}=\sqrt{k_{1} / m_{1}}, \quad \omega_{2}=\sqrt{k_{2} / m_{2}} ; \quad \xi_{1}=c_{1} / 2 m_{1} \omega_{11}$, $\xi_{2}=c_{2} / 2 m_{2} \omega_{2} ; \quad \chi=m_{1} / m_{2}, \omega_{1}, \omega_{2}$ - partial vibration frequency, $F_{c o n}\left(x_{1}-x_{2}\right)$ - contact interaction force, it simulates the impact. Initial conditions are:

$x_{1}(0)=0, x_{2}(0)=D, \dot{x}_{1}(0)=0, \dot{x}_{2}(0)=0$.

Numerical parameters of VIS are given in [9] and in Table 1 further.

\section{Impact softening by system parameters changing}

The clear criterion of impact rigidness or softness is absent. The typical trait of impact softness is its duration. Is it instantaneous or not? Let us examine the value - the coefficient of the relative impact duration $k_{c o n}=T / T_{\text {con }}$, where $T$ - period of external loading (1), $T=2 \pi \cdot \omega^{-1}$, $T_{c o n}$ - the time of impact i.e. the time of contact between bodies. The impact duration is $T_{c o n}=0,781 \mathrm{Mc}$ and the coefficient of relative impact duration is $k_{c o n}=T / T_{c o n}=1113,3$ for the motion with rigid impact. The bodies' displacements and exited contact force in this motion are shown at figures 4a,3a. Here VIS has numerical parameters that are given in Table 1.

Table 1. Numerical parameters of VIS with rigid impact.

\begin{tabular}{|l|c|c|}
\hline \multicolumn{1}{|c|}{ Bodies' characteristics } & Main body & $\begin{array}{c}\text { Attached } \\
\text { body }\end{array}$ \\
\hline Mass $m_{\mathrm{i}}, \mathrm{kg}$ & 1000,0 & 100,0 \\
\hline $\begin{array}{l}\text { Partial vibration frequency } \\
\omega_{\mathrm{i}}, \mathrm{rad} \cdot \mathrm{s}^{-1}\end{array}$ & 6,283 & 5,646 \\
\hline Young's modulus $E_{\mathrm{i}}, \mathrm{H} \cdot \mathrm{m}^{-2}$ & $2,1 \cdot 10^{11}$ & $2,1 \cdot 10^{11}$ \\
\hline Contact surface radius $R_{\mathrm{i}}, \mathrm{m}$ & 2 & 2 \\
\hline Coefficients $A, B, \mathrm{~m}^{-1}, q$ & $A=0,5 \quad B=0,5 q=0,319$ \\
\hline $\begin{array}{l}\text { External loading amplitude } \\
F_{0}, \mathrm{H}\end{array}$ & \multicolumn{2}{|c|}{500,0} \\
\hline $\begin{array}{l}\text { External loading frequency } \\
\omega, \mathrm{rad} \cdot \mathrm{s}^{-1}\end{array}$ & \multicolumn{2}{|c|}{7,23} \\
\hline
\end{tabular}

Let us examine what way can be chosen for decrease the coefficient of the relative impact duration $k_{c o n}$ (i.e. increase the impact duration $T_{c o n}$ ) by changing the parameters of VIS, in the main the stiffness parameters. For this problem decision we use numerical effective method based on theory and techniques of nonlinear programming for solution the problem of parameters optimization. We use the modified steepest descent method - gradient projection method with correction of residual with constraints $[9,13]$. We formulate the minimax problem like that: to find such parameters of VIS which will provide the smallest value of relative impact duration coefficient $k_{c o n}$, i.e. the largest value of impact duration $T_{c o n}$. We seek for the objective function $k_{c o n}=T / T_{\text {con }}$ by deciding the problem about steady-state exiting vibrations of VIS under the concrete system parameters. Numerical integration of motion equations (3) was fulfilled by Runge-Kutta 4th order method. The calculation of objective function gradient takes into account the constraints laid on the parameters. The obtained value determines the next optimization step. We investigated the influence of such parameters at impact duration: the attached body's mass $m_{2}$; the joining spring's stiffness $k_{2}$ (it comes into the partial frequency expression (3)); Young's modulus of both system bodies $E_{1}, E_{2}$; the radiuses of contact surfaces for both bodies $R_{1}, R_{2}$ in assumption that these surfaces are spherical (these radiuses comes into expressions of coefficients A and B in formula for Hertz's force (2)). The other parameters weren't changed. We have obtained such results. The impact duration was enlarged considerably under influence of these parameters changing. The graphics of displacements and contact force are shown at figure 5 for parameters values which are given in Table 2 .

Table 2. Numerical parameters of VIS with soft impact.

\begin{tabular}{|l|c|c|}
\hline \multicolumn{1}{|c|}{ Bodies' characteristics } & Main body & $\begin{array}{c}\text { Attached } \\
\text { body }\end{array}$ \\
\hline Mass $m_{\mathrm{i}}, \mathrm{kg}$ & 1000,0 & 310,0 \\
\hline $\begin{array}{l}\text { Partial vibration frequency } \\
\omega_{\mathrm{i}}, \text { rad }^{-1}\end{array}$ & 6,283 & 3,606 \\
\hline Young's modulus $E_{\mathrm{i}}, \mathrm{H} \cdot \mathrm{m}^{-2}$ & $1,4 \cdot 10^{10}$ & $1,0 \cdot 10^{6}$ \\
\hline Contact surface radius $R_{\mathrm{i}}, \mathrm{m}$ & 2 & 2 \\
\hline Impact duration $T_{\text {con }}, \mathrm{s}$ & $A=0,5 \quad B=0,5 q=0,319$ \\
\hline $\begin{array}{l}\text { Coefficient of impact } \\
\text { duration } k_{\text {con }}\end{array}$ & \multicolumn{2}{|c|}{8,87} \\
\hline
\end{tabular}

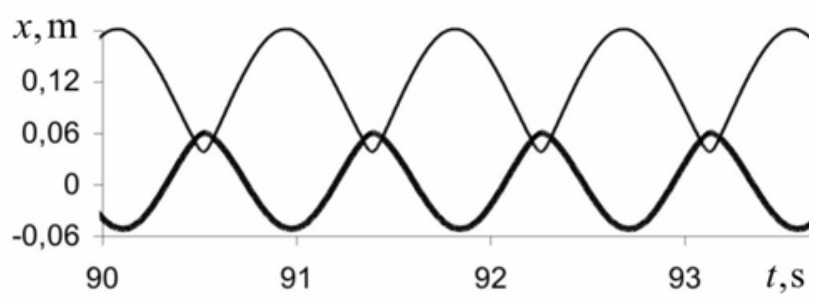

$F_{c o n}, \mathrm{\kappa H}$

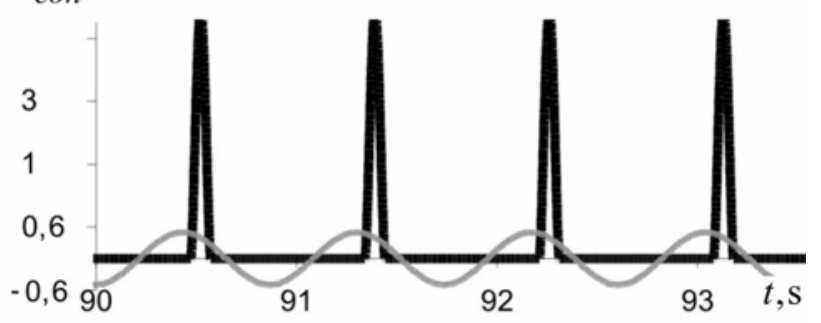

Fig. 5. Characters of $(1,1)$-periodical vibration regime

$$
\left(k_{\text {con }}=8,87\right) \text {. }
$$

At the displacement graphic (figure 5) we see how attached body (thin curve) penetrates into the main one (thick curve) due their local deformation. The contact force graphic (black curve) can be compared with the 
external loading graphic (grey curve). It isn't similar at "stick" already; it draws near the "bell".

The best result (i.e. the biggest value of impact duration) for $\mathrm{T}$-periodic one-blow vibration regime is shown at figure 6 .
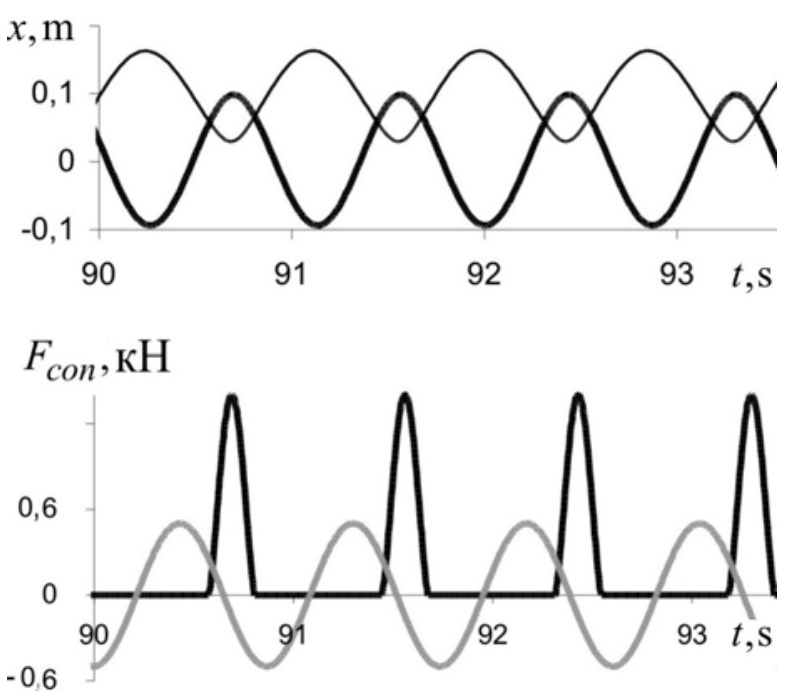

Fig. 6. Characters of $(1,1)$-periodical vibration regime $\left(k_{c o n}=3,73\right)$.

Let us notice some unexpected result obtained during researches: decrease of joining spring stiffness doesn't provide the considerable impact softening. Decrease of stiffness twice and ten times diminishes the contact impact force almost equally and practically doesn't increase the impact duration. Further decrease of stiffness causes the loss of stability for T-periodic one-blow regime and rise of nT-periodic multi-blow regimes. For further impact softening we can increase the impact duration some more when decreasing bodies' materials Young's modulus and changing the contact surfaces radiuses. But then the motion character changes too - motion becomes $n T$ periodic. Let us notice that the impact duration is increasing but the contact force is decreasing when the impact is becoming more soft.

\section{Conclusions}

The investigations fulfilled in this work allow to make the next conclusions.

- Parameters of VIS with rigid impact may be changed like that the impact will become the soft one. Gradient projection method with correction of residual with constraints gives the possibility to find the optimal system parameters which will provide the maximum impact duration.

- The using of nonlinear contact force based on quasistatic Hertz's theory allows simulating the impact in VIS with both rigid and soft impact $[14,15]$. Its using gives the possibility to find not only maximum value of contact force but its changing in time too, provides the calculation of impact duration. The using of this force provides also the diversified taking into account of the system parameters influence at its dynamic behaviour.

\section{References}

1. Babitsky V.I. Theory of Vibro-Impact Systems and Applications, Berlin: Springer-Verlag, (1998).

2. Ibrahim, Raouf A. Vibro-impact dynamics: modeling, mapping and applications. Vol. 43. Springer, (2009).

3. Ivanov, A. P. "Dynamics of systems with mechanical impacts." International Education Program, Moscow (1997): 336.

4. Blazejczyk-Okolewska, Barbara, Krzysztof Czolczynski, and Tomasz Kapitaniak. "Classification principles of types of mechanical systems with impacts-fundamental assumptions and rules." European Journal of Mechanics-A/Solids $\mathbf{2 3 . 3}$ (2004): 517-537.

5. Andreaus, Ugo, and Paolo Casini. "Dynamics of SDOF oscillators with hysteretic motion-limiting stop." Nonlinear Dynamics 22.2 (2000): 145-164.

6. Bazhenov V. A., Pogorelova O. S., Postnikova T. G., Goncharenko S. N. "Comparative analysis of modeling methods for studying contact interaction in vibroimpact systems." Strength of materials 41(4) (2009): 392-398.

7. Yue Ma, Manish Agarwal, Soumitro Banerjee Border collision bifurcations in soft impact system, Physics Letters A 354 (2006) 281-287.

8. Andreaus, Ugo, Bernardino Chiaia, and Luca Placidi. "Soft-impact dynamics of deformable bodies." Continuum Mechanics and Thermodynamics (2013): 1-24.

9. Bazhenov V. A., Pogorelova O. S., Postnikova T. G., Luk'yanchenko O.A. Numerical Studies of Dynamic Processes in the Vibroimpact Systems during Impact Simulation by the Contact Interaction Force, Strength of Materials 40(6) (2008) 656-662.

10. Bazhenov V. A., Pogorelova O. S., Postnikova T. G. Impact forces in vibroimpact systems, Strength of Materials and Theory of Structures 91 (2013): 13-28. (in Ukrainian)

11. Goldsmith W. Impact: The Theory and Physical Behaviour of Colliding Solids, Edward Arnold, London, (1960).

12. Machado, Margarida, et al. "Compliant contact force models in multibody dynamics: Evolution of the Hertz contact theory." Mechanism and Machine Theory 53 (2012): 99-121.

13. Gouliaev V.I., Bazhenov V. A., Koshkin V.L. Optimization Methods in structural mechanics.K.:UMK BO, (1988).-192 p. (in Russian)

14. .Bazhenov V. A., Pogorelova O. S., Postnikova T. G. Comparison of Two Impact Simulation Methods Used for Nonlinear Vibroimpact Systems with Rigid and Soft Impacts, Journal of Nonlinear Dynamics, vol. 2013, Article ID 485676, 12 pages, (2013). doi:10.1155/2013/485676.

15. Bazhenov V. A., Pogorelova O. S., Postnikova T. G. Dynamic behaviour analysis of different types vibroimpact systems. LAP LAMBERT Academic Publ. GmbH and Co. KG Dudweiler, Germany, (2013). (in Russian) 